\title{
Virtual Reality Models for the Preservation of the Unesco Historical and Artistical Heritage
}

\author{
Roberta Menghi ${ }^{1}$, Giuseppe Maino ${ }^{1,2}$, and Marianna Panebarco ${ }^{3}$ \\ ${ }^{1}$ Faculty of Preservation of the Cultural Heritage, University of Bologna, Ravenna site, \\ 5, via Mariani, Ravenna, Italy \\ giuseppe.maino@unibo.it, Roberta.Menghi@libero.it \\ ${ }^{2}$ ENEA, Italian National Agency for New Technologies, \\ Energy and Sustainable Economic Development, 4, via Martiri di Montesole, Bologna, Italy \\ giuseppe.maino@enea.it \\ ${ }^{3}$ Panebarco \& C., via Molino, 9, 48121 Ravenna \\ marianna@panebarco.it
}

\begin{abstract}
Reproduction of space with very high yield of photo-realism, through the use of special three-dimensional modelling techniques, enables the enhancement of the places of the "Great Mtskheta", the ancient capital city of Georgia, which are mostly in a state of extreme deterioration and abandonment. The production of integrated multimedia content to digital networks, is the ideal solution for a city like Mtskheta: A valid and concrete proposal so as to capture the added value of the opportunities created by the network, making the present reality.
\end{abstract}

Keywords: UNESCO, tourism, cultural heritage, 3D virtual world, Geographical Information System.

\section{Introduction}

More than 30 armed conflicts are currently ongoing around the world. Alongside the loss in human lives, more and more valuable heritage sites are turned into battlefield within the war theatres. More historic and archaeological patrimony is being vandalised, looted, illicitly traded spoiling UNESCO World Heritage Listed (WHL) historic city cores. Both the city of Byblos (Lebanon) and the city of Mtskheta (Georgia) - the focus of our project whose preliminary results are discussed in this paper - were just about to be severely affected by war operations respectively in 2006 the first and in 2008 the second. These events posed to the local administrations of both cities an additional concern, alongside that of the safety for the local civil community: Setting protective measures to protect the World Heritage Listed cities from destruction.

Both Lebanon and Georgia are parties of the 1954 Hague Convention (HC). The widespread destructions suffered by 'cultural properties' in addition to the huge loss of human lives during World War II, created the ground for the 1954 Hague Convention for the Protection of Cultural Properties during times of armed conflicts to be issued and ratified alongside its first protocol. 
The 1999 Second Protocol of the Convention entered into force in 2004 and in 2008 a Committee set at UNESCO finally issued the Guidelines for the implementation of the Convention. Over 56 years from its first ratification the HC remains widely neglected due to a lack of instruments for its implementation. In 2009 Guidelines for the implementation of the Convention and its two 1954 \& 1999 Protocols were issued and now we have conceived and developed a WAR FREE WORLD HERITAGE LISTED CITIES (WFWHLC) project to set a very concrete model in Byblos and Mtskheta, two cities that are patrimony of the humanity which were recently threatened by war.

This WFWHLC project is funded by the European Union within the framework of the CIUDAD program for a duration of 30 months and a budget of 540,740 Euro. Main project locations are Lebanon and Georgia and participants are the local governments of Jbail-Lebanon and Mtskheta-Georgia, WATCH - an NGO linked to UNESCO - in association with FOCUH (Turkey) and NEREA (Italy). NEREA is a joint venture of the University of Bologna and ENEA, Italian National agency for new technologies, energy and sustainable economic development. A first kick off meeting among the partners took place in Byblos, October 28-30, 2010.

The 1954 Hague Convention provides for the protection of 'cultural property' three moments, namely before, during and after armed conflicts; they coincide with three different approaches to be undertaken by national and/or international Cultural Heritage operators deployed in areas of conflict:

- $\quad$ Risk preparedness (and mitigation).

- Risk Management.

- Post conflict operations.

lt would be obvious that protective measures for the two cities should be inspired to the HC. However, one of the main contingencies faced by the concerned authorities in the implementation of the Convention and its Protocol is to be associated to the very limited availability of concrete case study that could serve as a reference when formulating their Risk Preparedness Plans (RPP) or when applying to UNESCO Committee for the Protection of Cultural Property in the event of armed conflict (Committee) for the introduction of protective measures. The subject requires a comprehensive approach as well as a solid policy for the maintenance of the protective measures during times of conflict. This implies the definition of a well designed pattern of relations between Civil and Military Authorities as well as the Civil Society at large and, first of all, the implementation of a multimedia database where all the information referring to the considered cities have to be stored and processed.

Based on these considerations WFWHLC intends to contribute to the definition of a comprehensive urban planning strategy that is designed to introduce and maintain the Enhanced Protection status for WHL cities. Therefore, overall objectives of our project are the development and applications of computer science tools in image and data processing in order (i) to create safety conditions for two WHL sites under immediate threat, (ii) to set good practice in the Urban Planning/Management of WHL cities under latent threat of armed conflicts, and (iii) to promote a widespread awareness about risks and risk mitigation measures needed to secure Enhanced 
Protection for WHL cities threatened by aimed conflicts, in accordance with international conventions.

Specific objective is to produce a Risk Preparation Plan and promote awareness about threats posed from armed conflicts for two WHL cities in support of the concerned authorities to candidate Byblos and Mtskheta for Enhanced Protection from UNESCO. Apart from the evident immediate effects produced by the project for the protection of Byblos and Mtskheta, the achievement of the project objectives will contribute to set several milestones, the most important of them being the methodological approach that will be disseminated to help other WHL cities that are experiencing the risks of conflicts and/or natural disasters without proper risk preparation and preventive plans.

Finally, major expected results include risk plans for the two WHL cities, risk mitigation policies in place, main actors, stakeholders and civil society awareness promoted by setting practices for the implementation of The Hague Convention (1954) Guidelines. The main tool to achieve these results is the implementation of a multimedia database where an open source geographical information system (GIS) is coupled to a digital platform for 3D interactive simulation of real world, freely available to the concerned and interested people. A preliminary analysis has been performed on Mtskheta city and is presented in this work.

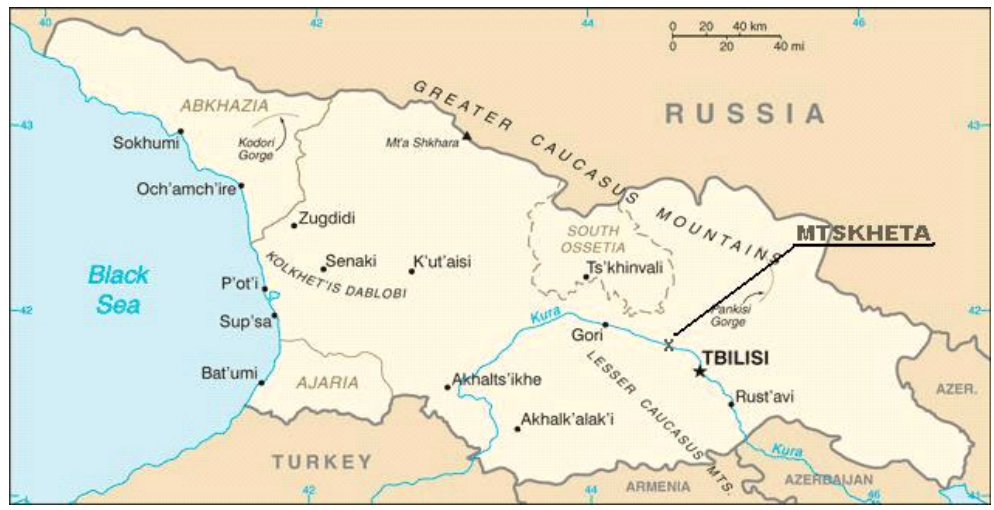

Fig. 1. Location of the city of Mtskheta

\section{Mtskheta Short History and Cultural Heritage}

In 1994 Mtskheta has joined the UNESCO World Heritage List for its historic medieval churches risen to one of the most significant examples of the early Christian architecture in Georgia, with a strong impact on the subsequent development of Georgian architecture. The document of the 18th session of the World Heritage held on 12 to 17 December 1994 in Phuket, Thailand states:

$<<$ The Committee, in inscribing this property on the World Heritage List, suggested to the State Party to change the name to "Historic Churches of Mtskheta (...) That this property be inscribed on the World Heritage List on the basis of criteria 
iii and iv: Criterion iii - the group of churches at Mtskheta bears testimony to the high level of art and culture of the vanished Kingdom of Georgia, which played an outstanding role in the medieval history of this region; Criterion iv - the historic churches of Mtskheta are outstanding examples of medieval ecclesiastical architecture in the Caucasus region $>>$.

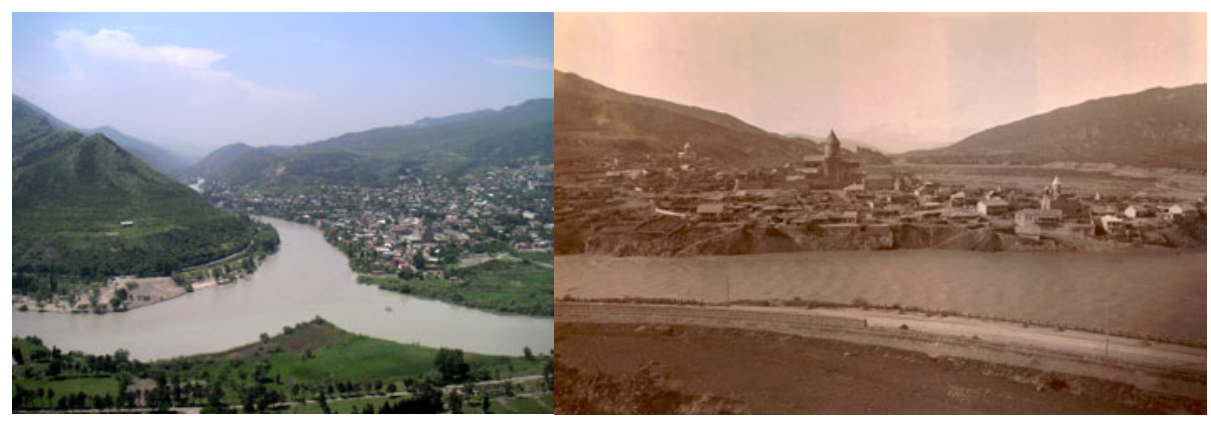

Figs. 2. \& 3. Different views of Mtskheta

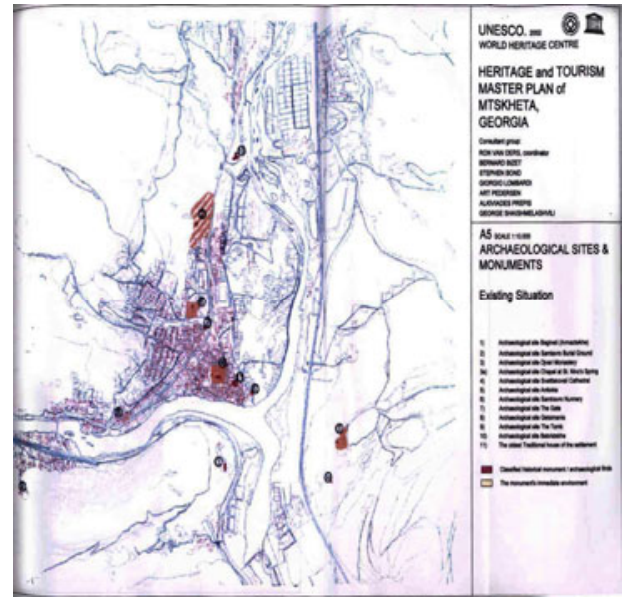

Fig. 4. Map of archaeological sites and monuments of Mtskheta $(1: 10,000)$, included in UNESCO World Heritage List in 1994

In 2005, the name of the property was changed and it was called the "Historical Monuments of Mtskheta". In 2009 it was included in the UNESCO list of Heritage in Danger, with the requirement from the state party to specify which monuments have been placed on the list. Actually, the National Agency for Cultural Heritage Preservation of Georgia works jointly with the UNESCO and re-nomination of monuments of Mtskheta is a possibility to address the problem.

In August 2008, the Russian army moved towards Tbilisi and reached environs of Mtskheta. Russian troops were in few kilometers from the city when the war was stopped due to the mediation by the President-in-Office of the European Union, 
French president Nicolas Sarkozy. The town of Mtskheta and its historical monuments remained intact during the conflict. However, a number of monuments in and around Tskhinvali region/South Ossetia were heavily damaged during the Russian-Georgian war.

Mtskheta is the ancient capital of Georgia, nestled in a picturesque setting located in a valley at the confluence of the rivers and Aragvi Mtkvari (figs.1-3) and is 20 / 25 $\mathrm{km}$ north-west from Tbilisi (now the capital of Georgia). From a moral point of view, Mtskheta has a formidable impact on the Georgians, as Masada was for the ancient Jews. The site is in fact a kind of inner sanctuary of artistic and religious culture of the country: Here, in 334 AD Georgians were converted to Christianity (according to history they were the second people to take the plunge, just four years after the neighboring Armenians) and Mtskheta, from III BC to V AD (see the archaeological sites depicted in fig.4) was the capital of the Kingdom of Georgia (even after the capital in the sixth century became Tbilisi, Mtskheta continued to be place of coronation and burial of the Georgian kings until the end of the Kingdom that took place in the nineteenth century). Christianity was then introduced in the fourth century in Mtskheta, and more generally in Georgia, by Saint Nino, and the first wooden church was built in the garden of the Royal Palace where now the cathedral of Svetitskhoveli stands (fig.5).

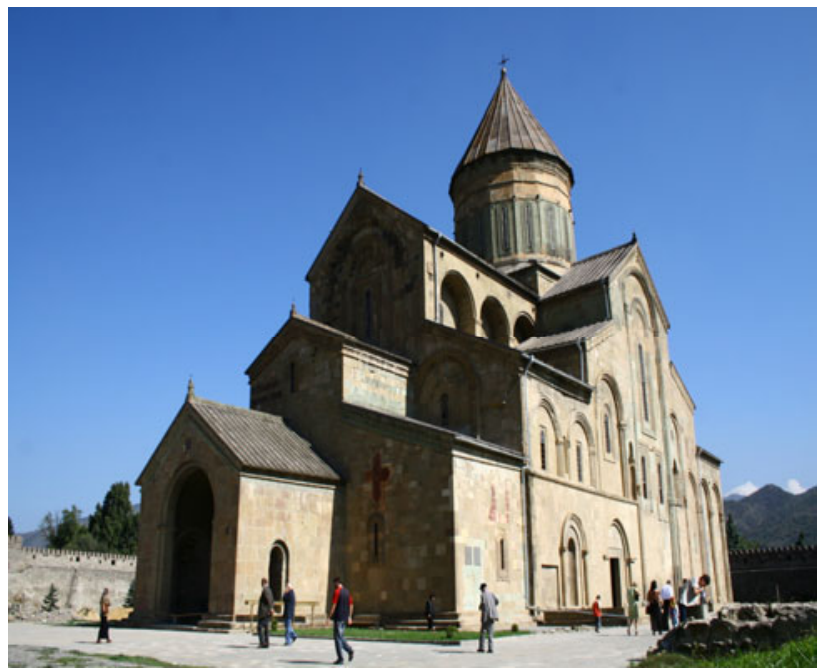

Fig. 5. The cathedral of Svetitskhoveli

Svetitskhoveli Cathedral (XI century) and Djvary Monastery (IV century) are still major landmarks, symbols of the country, together with the fortress of the Acropolis of Bebris Tsikhe, built by Armaz Tsikhe in the III century. The city is still the seat of the autocephalous Georgian Orthodox Apostolic Church: It has therefore retained a central role as a religious center of the country and home to the Katolicos (later elevated to Patriarch), an important reference point for the Georgians and the believers in other parts of the world. 
Despite the cultural importance and the many churches (essential for the development of medieval architecture in the Caucasus), the modern city in appearance is rather modest, both in terms of the size and architecture of today's buildings. An added value of the place lies in its natural arena, the environment particularly impressive that can be viewed from various sites, providing a smooth, pristine landscape that contributes to the small size of the city and to its architecture that blend in harmony with nature.

Moreover, because of the serious condition and, in some cases (e.g., the Getsimania church), abandonment of some monuments and recent improvident works of 'restoration' carried out which have partially eliminated the historic features of the monuments, most of the archaeological sites has not any form of protection, simply a few ways to shed and fence protection. In the case of the Necropolis of Samtvro which has a simple roof to repair a large area of great historical and artistic value from the weather, existing facilities are clearly insufficient to protect the sites from the natural elements, but also from possible theft or damage caused by man.

A significant example of wrong intervention according to the UNESCO Commission is given by the present situation of the above-mentioned Svetitskhoveli Cathedral, where the ecclesiastical authorities have carried out irreversible restorations, by the use of reinforced concrete and by removing important ornaments of the cathedral, with no previous agreement with local authorities and violating the International Convention for the protection of UNESCO:

$<<(\ldots)$ despite its great importance, it is now in imminent danger due to the largescale interventions of the local church authorities. These interventions, which have been carried out on the authorities' own initiative in the name of the monastery's current operational needs without any control by the local or central services responsible, are beyond any scientific ethics concerning protection of and respect for monuments and they are in blatant violation of UNESCO's international protection convention >>

The Committee of UNESCO has formally asked Georgia to start a serious work of recovery and maintenance, given the alarming situation in terms of the various historic buildings:

$<<(\ldots)$ the state of conservation of the archeological components of the World heritage property, their progressive deterioration and the abandonment of the conservation efforts by the state party, nothing that this loss has a major impact on the outstanding universal value, authenticity and the integrity of the property and further urges the state party to develop a special program on protection of all archeological components $>>$.

In order to "remove" Mtskheta and its monuments from the list of monuments in danger, a fundamental aspect is the (reborn) awareness of local political authorities with regard to maintenance necessary for the preservation of the heritage of the city, although there are difficulties in involving all stakeholders in the management. $<<$ The establishment of the UNESCO and International Relations Unit at the National Agency for Cultural Heritage Preservation and the establishment of the State Commission for World Heritage are indicators to the achieved progress $>>$. 
In this respect, GIS archives and 3D simulations of virtual reality may represent a valuable help for local authorities, restorers and scholars like art historians and archaeologists to perform these duties.

\section{Mtskheta Virtual World}

What is 3D? Essentially, a form of representation of space in its three dimensions on a two-dimensional plane. The 3D technology is expanding for years with a trend of exponential growth. An increasing number of sectors that use the techniques of threedimensional reconstruction: From aeronautics to medicine, from biology to chemistry, from cinema to television.

In the cultural heritage sector it has been widely used for 3D reconstruction of places that no longer exist or have been partially destroyed; many are the examples of reconstructions of archaeological sites, thanks to the guidance of scholars and art historians. But the 3D can also be used as a tool to conserve, preserve and promote cultural heritage. More and more repositories in the future will spread out in threedimensional objects, buildings, monuments and archaeological sites will be associated not only to descriptive text files and images but also to detailed three-dimensional documents available at several levels.

The use of 3D will represent a rich and exciting development of archives: The cataloguing of objects, buildings and monuments in the future will necessarily include, in addition to traditional data and details (see cards for works of art) also a further field that will contain a link to the three-dimensional reconstruction, useful for the scholar to the architect, for the student to the restorer, for the curious ...

A virtual world is a place simulated by computer graphics, where users - connected to the Internet - interact via avatars. The term comes from Sanskrit and Hindu mythology and religion, and means taking a physical body by God. An avatar is a digital representation of the user who enters a virtual world. The most popular virtual worlds include MMORPGs (Massively Multiplayer Online Role-Playing Game, online games that simultaneously connect thousands of people around the world). What are the main features of a virtual world?

Sharing experiences: Multiple users can, everyone at her/his desk, log on simultaneously in the world;

real time: The user decides to "live" what to do, how to get around, where to go and everything happens at the very moment in which the actions are carried;

interactive and participatory creation: The user can click on objects, modify them, build new ones;

socialization: Users can socialize and create groups and communities through various communication channels;

persistence: The world exists regardless of whether users are connected;

involvement: The three-dimensionality and esplorabilità the digital space, combined with interactivity and allow a greater degree of social interaction and involvement. 
We present a brief description of VirtualLife, an experimental platform whose development is currently in progress, and is the subject of a project co-financed by the European Union under the Seventh Framework Programme - ICT. The aim of the consortium that develops VirtualLife (9 partners from 9 different EU countries, including 7 universities and $2 \mathrm{SME}$ ) is the creation of a virtual world platform innovative in terms of both technical and philosophical items, that solves some of the main limitations of existing parallel universes. The research teams of VirtualLife are focusing more on administrative and legislative aspects and on security and data protection.

Basically the project aims to create an immersive virtual environment based on a peer-to-peer network (which technically provides benefits primarily on the distribution of computational load) and where the many capabilities offered by the programming language are combined with the reliability of a secure communications infrastructure, dedicated not only to entertainment but also to socialization, training and business.

One of the main aims of the project is thus to create, in a collaborative way, the first Virtual Constitution, for the creation and administration of the new virtual nation that will come to be absolutely necessary to safeguard the common interests of citizens and private virtual visitors. This opportunity opens the way for the creation of immersive visits in real time of the various archaeological sites scattered in the valley where it stands Mtskheta, visits that can be used by remote users connected to Internet broadband. The project is targeted to the global market, which intercepts the growing demand of the people's network of rich, charming and engaging experiences, so extending the visibility of Mtskheta and its heritage.

Working in this direction will seize a number of advantages:

The overall visibility and global, with the 3D modeling of heritage and its dissemination on line, will help to increase the knowledge of monuments, places and archaeological sites that are now neglected, in a manner that seriously compromises their survival (especially those outside the perimeter of the city). Only through the dissemination of knowledge we can preserve our heritage.

Formation of a three-dimensional archive, the creation of a database of threedimensional digitized documents, represents for Mtskheta (but not only for this town) the opportunity to recreate the perception of the environment, simulating the look of the place of the old "residents." This is a resource, potentially limitless, which remains "forever" to a past, whose existence will be passed on to future generations.

New perspectives of knowledge, using a multi-dimensional dynamic management for Mtskheta and its heritage, can be developed for each archaeological site, an apparatus of multimedia (eg, cards, audio, video) and information data to emphasize or devise routes that provide movements and points of view impossible in the real world, so as to return to each of the items emphasizing in particular interesting anecdotes and stories, explaining the context in which the work was carried out (interestingly, in these cases, it is also the approach to virtual restoration). A captivating experience that can arouse emotions in the user, such as a visit by the "fly" to match the decor (for example, the frescoes in the dome of the cathedral, the complex of Djvari) in real time and absolute freedom. By this way the visitor has a virtual unique and unforgettable experience. All this information is also useful for those planning a trip to Georgia and also for those who, once back home, will 
reinforce what they admired in the just concluded trip, taking advantage of opportunities that in the physical world would be a complex mechanism for cost and logistical problems.

Economic benefits, the development of multi-dimensional, against an initial investment due to the cost of modeling only allows the worldwide visibility, greater knowledge of the city, thus possibly leading to an increase in tourism and foreign investment: Revenues may be, in part, provided to the agencies in the restoration and establishment of appropriate structures for the protection of archaeological sites located on the whole area.

It should be emphasized that an experience like this, and more generally the application of virtual reality methods to heritage, in this case the city of Mtskheta, must not be regarded as a debasement of the real assets, but rather an added value and valuable incentives for the conservation, preserving the existence of places, buildings and other property protected by a disclosure difficult to achieve with the "traditional" media (especially in those areas, such as Georgia, suffering from general security problems, due to the turbulent past / present). Just the transformation of the historic and artistic heritage of Mtskheta, opened to the world, cosmopolitan, through virtual tours, would be able to promote a collective cultural growth, as a guarantee "for the safety" of the precious heritage preserved here, property of humanity, in fact.

In the specific case of Mtskheta it could be envisaged to achieve the following 3D locations: Hypothetical reconstructions of sites partially destroyed due to severe neglect and the "turbulent" past of the city of Mtskheta. Some examples include the site of the Valley Armatsikhe, the acropolis of Mtskheta once ancient royal residence for centuries, thus placing virtual sites of origin of objects that are currently in the State Museum of Janashia and in the ethnography and archeology museums of Mtskheta, as well as all the archaeological finds discovered in the tombs of the Necropolis of Samtavro and Armatiskhe, the area where once stood the residence of King Pitiakhshi.

Other actions include possible reconstructions of finds missing or partially destroyed, the ability to create virtually murals, frescoes, paintings that have disappeared with the passage of time or whose conditions are so degraded as not to allow the vision; a significant example is in the village of Dzalisi, not far from Mtskheta, the mosaic floor, known as "The House of Dionysos", dating from the third century. The Svetitskhoveli cathedral has undergone several restorations over the centuries inside, that have eliminated many of the frescoes by altering the original appearance. There the mode of "flight" can be used in real time and absolute freedom; Svetitskhoveli cathedral is richly painted inside with exceptional frescoes dating from the seventeenth century, depicting scenes from the Gospel and the Old Testament, episodes of the Conference of Kartli, portraits of historical figures.

Reconstructions are made possible of the various periods of development of a complex site, as the Monastery of Djvary that includes several buildings constructed at different times, moreover giving the user the ability to move easily from one level to another time and to compare the configurations of the site at various times.

Objectives are the overall visibility and global formation of a three-dimensional archive; new perspectives of knowledge; economic benefits. The project aims to achieve a broad consensus from potential users such as different tourist segments; circuit of the school network; professionals. 


\section{Vectorial GIS Supporting 3D Virtual Reality Models}

Quantum GIS (QGIS) is a user friendly Open Source Geographic Information System (GIS) licensed under the GNU General Public License. QGIS is an official project of the Open Source Geospatial Foundation (OSGeo). It runs on Linux, Unix, Mac OSX, and Windows and supports numerous vector, raster, and database formats and functionalities. Quantum GIS provides a continously growing number of capabilities defined by core functions and plugins. You can visualize, manage, edit, analyse data, and compose printable maps. The major features include:

View and overlay vector and raster data in different formats and projections without conversion to an internal or common format;

create maps and interactively explore spatial data with a friendly graphical user interface;

create, edit and export spatial data using digitizing tools for GRASS and shapefile formats, the georeferencer plugin, GPS tools to import and export GPX format, convert other GPS formats to GPX, or down/upload directly to a GPS unit;

perform spatial analysis using the fTools plugin for Shapefiles or the integrated GRASS plugin, including map algebra, terrain analysis, hydrologic modeling, network analysis, and many others;

publish maps on the internet using the export to Mapfile capability.

In addition, our research group utilizes GRASS GIS (Geographic Resources Analysis Support System) that is an open source, free software Geographical Information System (GIS) with raster, topological vector, image processing, and graphics production functionality operating on various platforms through a graphical user interface and shell in X-Windows. It is released under GNU General Public License (GPL). GRASS contains over 350 programs and tools to render maps and images on monitor and paper; manipulate raster, vector, and sites data, process multi spectral image data and create, manage, and store spatial data.

Originally developed by the U.S. Army Construction Engineering Research Laboratories (USA-CERL, 1982-1995), a branch of the US Army Corp of Engineers, as a tool for land management and environmental planning by the military, GRASS has evolved into a powerful utility with a wide range of applications in many different areas of scientific research.

\section{Conclusions and Perspective Work}

The three-dimensional development of the cultural heritage by creating a parallel world of multi-dimensional goods integrated to telematic networks does not want to replace the physical model, but rather making it possible to support it with the provision of services otherwise too expensive or even impossible.

The places, squares, monuments, museums, mansions, palaces, churches and all the excellence of the artistic and historical heritage, are to most people silent witnesses of their own history. Through their three-dimensional processing, digital clones, it is technically possible let them interact, through the development of open and dynamic content that will deepen their story, giving the users the feeling of a "live" presence. In addition to the perspectives outlined above, it is interesting to mention the 
development of other applications, which are present not only in situ and remotely accessible. We refer to the installation of multimedia interactive stations (totems), scattered within archaeological sites. These are very powerful communication tools, dynamic and flexible enough to guide the visitor on a tour of "increased" reality and full of content (with the possibility of hypothetical reconstructions of the site, etc..), through the use of various interactive techniques such as video, keyboard and touch screen.

The use of guides on handheld devices, mobile guides, are able to deepen and complete the travel experience of the user, providing the opportunity to take guided tours or access to detailed information concerning the site or relating to specific items on display (see the many, and unknown graves in the Necropolis of Samtavro). The use of mobile guides would also be useful to the visitor during the trip, providing useful information about routes and directions to take (given also the scarcity of signs throughout the area of Mtskheta). Also it is worth mentioning a final interesting aspect of mobile guides, which deal with multi-users to promote social interaction among visitors: The multi-user visits and collaborative group is a key to a successful learning environment.

The Network and the three-dimensional shapes of the real world where you can create new scenarios assume the building of parallel worlds free from physical laws, against initial investment and operating costs very low. Far from thinking of the latest technology as a substitute, it means a direct and personal dialogue with artistic and historical assets. We turn to this as a means of knowledge aimed at overcoming the limits of sense perception, identifying possible solutions to be adopted for the radical change in information technology, without having to invest too many energy, resources, time, learning tools, which require skills of other professional profiles $>$.

The methods of virtual reality considered in this work to take advantage of multimedia for planning and creating a rich calendar of events parallel to the normal activities in the physical world, easily fit into a broader discussion of preservation and enhancement, decline in contexts where the infinite dimension "virtual" protect and preserve the assets which are also at risk (in most cases due to human intervention).

\section{References}

UNESCO, 18com XI-inscription: The City-Museum Reserve of Mtskheta, Georgia (1994), http: / / whe. unesco.org/en/decisions/3217

UNESCO, 33COM 8C.1 - Update of the List of World Heritage in Danger (2009), http: / /whc.unesco.org/en/decisions/1983

VirtualLife (2010), http: / / www . ict-virtuallife.eu

KUNSTHISTORICHES INSTITUT in FLORENZ, Church of Jvari, Cathedral of Svetitkhiveli,

http://expo.khi.fi.it/galleria/georgia/katli/

chiesa-di-javari-mtskheta

http://expo.khi.fi.it/galleria/georgia/katli/

cattedrale-di-svetitskhoveli-mtskheta

QGIS: http: / /www.qgis.org

GRASS: http://grass.bologna.enea.it 